\title{
CONVERGENCE RATES IN THE LAW \\ OF LARGE NUMBERS
}

\author{
BY LEONARD E. BAUM AND MELVIN KATZ
}

Communicated by W. Feller, July 5, 1963

1. Let $\left\{X_{i}: i=1,2, \cdots\right\}$ be a sequence of independent and identically distributed random variables and let $S_{n}=\sum_{k=1}^{n} X_{k}$. Recently a number of papers [1], [2], [3] and [5] have considered the problem of relating the existence of moments of the $X_{k}$ 's with the convergence of series of the form $\sum_{n=1}^{\infty} n^{t-2} P\left\{\left|S_{n}-E S_{n}\right|>n \epsilon\right\}$, where $t \geqq 1$ and $\epsilon>0$.

The purpose of this note is to present results that extend, strengthen and complete the previous work done on this problem.

2. It is known that for $t \geqq 1$ one has $E\left|X_{k}\right|^{t}<\infty$ and $E X_{k}=\mu$ if and only if $\sum_{n=1}^{\infty} n^{t-2} P\left\{\left|S_{n}-n \mu\right|>n \epsilon\right\}$ for all $\epsilon>0$. Since $t \geqq 1$, this theorem concerns sequences of independent and identically distributed random variables for which the Strong Law of Large Numbers holds and thus it is natural to study the rate of convergence to zero of the sequence $\left\{P\left[\sup _{k \geqq n}\left|\left(S_{k}-E S_{k}\right) / k\right|>\epsilon\right], n=1,2, \cdots\right\}$ as well as the sequence $\left\{P\left[\left|S_{n}-E S_{n}\right|>n \epsilon\right], n=1,2, \cdots\right\}$. The determination of the rate of convergence to zero of the first sequence is completely solved by the following two theorems. In everything to follow $\left\{X_{k}: k=1,2, \cdots\right\}$ will denote a sequence of independent and identically distributed random variables.

THEOREM 1. Let $t>1$. The following three statements are equivalent:

(a) $E\left|X_{k}\right|^{t}<\infty$ and $E X_{k}=\mu$,

(b) $\sum_{n=1}^{\infty} n^{t-2} P\left\{\left|S_{n}-n \mu\right|>n \epsilon\right\}<\infty$ for all $\epsilon>0$,

(c) $\sum_{n=1}^{\infty} n^{t-2} P\left\{\sup _{k \geqq n}\left|\left(S_{k}-k \mu\right) / k\right|>\epsilon\right\}<\infty$ for all $\epsilon>0$.

Note that in this theorem $t$ is greater than one. For $t=1$ it is true that (a) and (b) are equivalent, however, (c) is not implied by either of these conditions. For $t=1$ in (c) of the above theorem the correct equivalent conditions are given by the next theorem.

THEOREM 2. The following three statements are equivalent:

(a) $E\left|X_{k}\right| \lg ^{+}\left|X_{k}\right|<\infty$ and $E X_{k}=\mu$,

(b) $\sum_{n=1}^{\infty} n^{-1} \operatorname{lgn} P\left\{\left|S_{n}-n \mu\right|>n \epsilon\right\}<\infty$ for all $\epsilon>0$,

(c) $\sum_{n=1}^{\infty} n^{-1} P\left\{\sup _{k \geqq n}\left|\left(S_{k}-k \mu\right) / k\right|>\epsilon\right\}<\infty$ for all $\epsilon>0$.

The next two theorems determine rates of convergence when the sums $S_{n}$ are normed by $n^{\alpha}, \alpha>1 / 2$, and when the first moment may 
not exist but $E\left|X_{k}\right|^{t}<\infty$ for $0<t<1$. In particular, Theorem 1 is a special case of Theorem 3 but it has been singled out since it is the "classical" and most interesting case.

TheOREM 3. Let $t \geqq 1, r>1$ and $1 / 2<r / t \leqq 1$. The following three statements are equivalent:

(a) $E\left|X_{k}\right|^{t}<\infty$ and $E X_{k}=\mu$,

(b) $\sum_{n=1}^{\infty} n^{r-2} P\left\{\left|S_{n}-n \mu\right|>n^{r / t} \epsilon\right\}<\infty$ for all $\epsilon>0$,

(c) $\sum_{n=1}^{\infty} n^{r-2} P\left\{\sup _{k \geqq n}\left|\left(S_{k}-k \mu\right) / k^{r / t}\right|>\epsilon\right\}<\infty$ for all $\epsilon>0$.

TheOREM 4. Let $t>0, r>1$, and $r / t>1$. The following three statements are equivalent:

(a) $E\left|X_{k}\right|^{t}<\infty$

(b) $\sum_{n=1}^{\infty} n^{r-2} P\left\{\left|S_{n}\right|<n^{r / t} \epsilon\right\}<\infty$ for all $\epsilon>0$,

(c) $\sum_{n=1}^{\infty} n^{r-2} P\left\{\sup _{k \geqq n}\left|S_{k} / k^{r / t}\right|>\epsilon\right\}<\infty$ for all $\epsilon>0$.

Finally we wish to comment that the proofs of the known results in this area can be considerably simplified and unified. In particular, it is not necessary to give a special proof for the result in [5] that $E X_{k}=\mu$ if and only if $\sum_{n=1}^{\infty} n^{-1} P\left\{\left|S_{n}-n \mu\right|<n \epsilon\right\}<\infty$ for all $\epsilon>0$.

These details as well as the proofs of the above theorems will appear elsewhere.

\section{REFERENCES}

1. P. Erdös, On a theorem of Hsu and Robbins, Ann. Math. Statist. 20 (1949), 286291.

2. P. L. Hsu and H. Robbins, Complete convergence and the law of large numbers, Proc. Nat. Acad. Sci. U.S.A. 33 (1947), 25-31.

3. M. L. Katz, The probability in the tail of a distribution, Ann. Math. Statist. 34 (1963), 312-318.

4. M. Loève, Probability theory, Van Nostrand, New York, 1960.

5. F. Spitzer, A combinatorial lemma and its application to probability theory, Trans. Amer. Math. Soc. 82 (1956), 323-339.

Institute for Defense Analyses 